\title{
Cardiac Surgery: Current Status of Aortic Valve Replacement
}

\author{
Tarek Malas $^{1 *}$, Cesario Bianchi ${ }^{2}$ and Marc Ruel ${ }^{1}$
}

${ }^{1}$ Division of Cardiac Surgery, University of Ottawa Heart Institute, 40 Ruskin Street, Ottawa, Ontario, Canada

${ }^{2}$ Department of Surgery, Rhode Island Hospital, Brown University, Providence, Rhode Island 02912, USA

\begin{abstract}
Aortic Stenosis (AS) is present in approximately $2 \%$ of people and is most commonly caused by degenerative calcification of the aortic valve. Without aortic valve intervention, aortic stenosis is associated with substantial morbidity and mortality in patients, particularly in those presenting with angina, syncope, or heart failure.

The current gold standard for treatment of Aortic Stenosis is surgical aortic valve replacement, most commonly with either a mechanical or a bioprosthetic aortic valve. The choice of valve prosthesis is dependent on a myriad of factors involving patient preferences, age, life expectancy, presence of other comorbidities, and contraindications to lifelong anticoagulation. Although surgeons have traditionally recommended mechanical valves for patients younger than 60 for their long-term durability, studies have demonstrated that there was no significant difference in long-term survival for tissue versus mechanical aortic prosthesis for patients younger than 60 years.

A large proportion of patients deemed high-risk for surgical valve replacement have been successfully treated with percutaneous deployment of an aortic valve by Transcatheter Aortic Valve Implantation (TAVI) technologies in a less invasive method. TAVI devices have shown immense success in the clinical realm via both the transfemoral and transapical approaches. The PARTNER trial investigators have demonstrated in their first cohort of patients that transfemoral TAVI was superior to standard medical therapy in reducing any-cause mortality, cardiovascular mortality, and repeat hospitalization. Furthermore, a comparison of TAVI to surgical aortic valve replacement (SAVR) in specific high-risk populations of patients in the PARTNER trial demonstrated non-inferiority of TAVI compared to SAVR. While TAVI has shown success in a large group of patients, its limitations and its long-term outcomes are being explored in a range of studies. Currently, surgical AVR is still the gold standard for treatment of aortic stenosis, but percutaneous $\mathrm{TAVI}$ technology continues to play a key role in management of aortic stenosis in certain subpopulations of patients.
\end{abstract}

\section{Keywords: Aortic valve; Aortic stenosis; Transcatheter}

\section{Review}

Aortic Stenosis (AS) is the most common cardiac valve pathology and is prevalent in approximately $2 \%$ of people 65 years and older [1]. Two factors account for the common occurrence of aortic stenosis-increasing age and the presence of bicuspid aortic valves [2]. Bicuspid Aortic Valves (BAV) are prone to stenosis and are found in approximately $1-2 \%$ of the population. Studies have suggested that BAV have a significant incomplete genetic inheritance pattern with influence from non-genetic factors as well [3,4]. Aortic stenosis (AS) is a major cause of morbidity and mortality in patients - prognosis is poor for patients presenting with angina, syncope, or heart failure [2]. However, medical therapy alone is not effective for the treatment of AS [1]. The current gold standard for treatment of AS is surgical aortic valve replacement.

Aortic Stenosis is most commonly caused by degenerative calcification, proliferative and inflammatory changes via lipid accumulation, up-regulation of angiotensin-converting enzyme activity and infiltration of macrophages and T-lymphocytes [5]. Risk factors for Aortic Stenosis are similar to those of atherosclerosis - elevated LDL cholesterol and lipoprotein A, diabetes, smoking, and hypertension. Furthermore, aortic valve sclerosis is associated with higher risks of cardiovascular death and myocardial infarction [5]. This is due to progressive left ventricular outflow obstruction secondary to increased afterload. While the response of gradual development of left ventricular hypertrophy is adaptive in maintaining cardiac output, this increased myocardial mass has poorer coronary reserve and diastolic and systolic dysfunction ensues [2]. Aortic Stenosis perturbs the aortic valve's passive mechanism that allows blood to flow with minimal pressure differences between the ventricle and aorta [5]. Consequently, patients develop signs and symptoms of angina, dyspnea, and congestive heart failure.

Patients generally do not develop symptoms of aortic stenosis until the valve area is reduced to approximately $25 \%$ of its original size. Aortic valve replacement is indicated when patients develop severe symptomatic AS. Guidelines categorize severity of AS by AV area and mean gradients, respectively: $1.5 \mathrm{~cm}^{2}$ and $<25 \mathrm{mmHg}$ for mild, $1.0-1.5 \mathrm{~cm}^{2}$ and $25-40 \mathrm{mmHg}$ for moderate, and $<1.0 \mathrm{~cm}^{2}$ and $>40 \mathrm{mmHg}$ for severe AS [6]. On average, patients develop symptoms when the AV area approaches 0.6 to $0.8 \mathrm{~cm}^{2}$ [7]. In addition, surgical replacement is warranted when patients with severe aortic stenosis require concomitant cardiac or aortic surgery or develop LV systolic dysfunction defined as an ejection fraction less than 50\% [6]. However for patients who do not undergo aortic valve replacement, their life expectancy approaches 5 years with symptoms of angina, 3 years with syncope, and 2 years with the advent of congestive heart failure [2]. Excessive waiting periods with symptomatic AS lead to increased mortality and sudden death [8]. Controversies exist for aortic valve replacement in severe asymptomatic AS and in other scenarios, but this will not be addressed in this article.

The ideal valve for Aortic Valve Replacement (AVR) should have minimal residual transaortic pressure gradients and should be durable, affordable, highly thrombo-resistant, simple to implant, and widely available [5]. Unfortunately, this valve does not exist with current technology [9]. There are two main classes of prosthetic aortic valvesmechanical and bioprosthetic valves. Other less commonly used

*Corresponding author: Tarek Malas, Division of Cardiac Surgery, University of Ottawa Heart Institute, 40 Ruskin Street, Ottawa, Ontario, K1Y 4W7, Canada E-mail: tmalas@ottawaheart.ca

Received June 18, 2012; Accepted July 23, 2012; Published July 24, 2012

Citation: Malas T, Bianchi C, Ruel M (2012) Cardiac Surgery: Current Status of Aortic Valve Replacement. Surgery S3:001. doi:10.4172/2161-1076.S3-001

Copyright: $\odot 2012$ Malas T, et al. This is an open-access article distributed under the terms of the Creative Commons Attribution License, which permits unrestricted use, distribution, and reproduction in any medium, provided the original author and source are credited. 
bioprosthetic valves include stentless heterograft bioprosthetic valves, allograft valves, and pulmonary allograft valves. This group of valves has exceptional physiologic and hemodynamic properties and is less prone to thrombosis and endocarditis [5]. However, their use is limited due to availability, the technical difficulty of implantation, and poorer long-term durability. Bioprosthetic valves are composed of bovine or porcine pericardial tissue and are fixated with glutaraldehyde to crosslink collagen fibers, enhance durability, and reduce antigenicity of the foreign tissue [5]. Unlike bioprosthetic valves, mechanical valves are composed of materials ranging from Stellite to Silastic, which necessitates lifelong anticoagulation. In addition to an enhanced effective orifice area, mechanical valves boast long-term durability superior to that of bioprosthetic valves. Although mechanical valves have a much lower risk of re-intervention, they are more prone to thrombotic complications compared to bioprosthetic valves [7]. However, a study by Hammermeister et al. [10] comparing long-term outcomes of AVR with bioprosthetic or mechanical valves revealed no difference in thromboembolic events for patients on anticoagulation. In a prospective study at our center which evaluated stroke rates in a cohort of 3,189 patients undergoing aortic and/or mitral valve replacement, Ruel et al. [11] found that patients with a mechanical valve in the mitral position had 1.24 times the risk of embolic strokes compared to those with bioprosthetic valves. However, there was no observed difference between mechanical and bioprosthetic valves in the aortic position. This finding may be explained by the high-velocity and low-stasis profile found in the aortic position as compared to that of the mitral. However, major bleeding events-those requiring hospitalization or transfusion, of intracranial location, or causing mortality - were significantly higher in mechanical prostheses versus bioprostheses in both valve positions.

The decision for the type of prosthesis is complex and must take into account patient preference as well as a myriad of factors related to the characteristics of the prostheses. This involves patient age, life expectancy, presence of comorbidities, and indications or contraindications for anticoagulation use. In general, mechanical valves may be implanted for patients without any contraindication to longterm anticoagulation and with low risk for accelerated bioprosthetic deterioration (young age, hyperparathyroidism, or renal insufficiency) [9]. This also includes patients with metabolic syndrome, which has been recently verified as an independent risk factor for accelerated valve deterioration by Briand et al. [12]. On the other hand, patients who are poorly compliant with medication, have contraindications to anticoagulation, have limited life expectancy, or are of childbearing age, may benefit from a bioprosthetic valve. There are only 2 large randomized control trials that compare survival rates and valve-related complications for mechanical versus bioprosthetic valves. These studies were limited because outcomes were not based on different age groups, follow-up was limited, and a large proportion of patients had previous redo thoracotomy/sternotomy prior to a first AVR [13].

In general, multiple centers recommend bioprosthetic valves for patients older than 60-70 from outcome data analysis [14-16]. This is because bioprosthetic valves deteriorate more rapidly at a younger age; the Veterans Affair trial showed structural deterioration of more than $26 \%$ of patients for ages $>65$ and only $9 \%$ for ages $<65$ in a 15 year outcome analysis [10]. Furthermore, with the increasing life expectancy of patients, the proportion of patients with bioprosthetic valve deterioration will likely rise. The age of 70 is perceived as a transitional point for choice of aortic valve prosthesis wherein surgeons must take into account factors that determine long-term outcomes $[17,18]$. Surgeons have traditionally recommended mechanical valves for patients younger than 60 for their long-term durability [5] However, this is not well supported by data for patients younger than 60 years $[19,20]$. In a 20 -year cohort conducted at our center, Ruel et al. [13] demonstrated that there was no significant difference in longterm survival for tissue versus mechanical single-valve prosthesis for patients younger than 60 years. Long-term outcomes were similar despite a higher reoperation rate in bioprosthetic implants. At 20 and 25 years, survival was $65.5 \pm 3.2 \%$ and $51.7 \pm 4.8 \%$, respectively, in AVR patients with a tissue bioprosthesis, and $52.3 \pm 4.4 \%$ and 41.2 $\pm 5.2 \%$, in those with a mechanical prosthesis [13]. Furthermore, subgroup analysis for patients less than 50 years of age at initial AVR also revealed no significant difference in survival (HR: 0.8 , initial AVR tissue versus mechanical in adults less than 50 years old; $95 \%$ CI: 0.5 , $1.2 ; P=0.3$ ) (Figure 1).

The results of this study suggest that mechanical prostheses are not necessarily warranted for younger patients, contrary to recommendations in the literature. There is an increasing trend towards the use of bioprosthetic valves in North America. One important factor for this increase is due to improvements in prosthetic valve durability compared to older generation products [18]. Multivariate analysis in our study revealed that patient age, the presence of coronary artery disease, earlier year of surgery, and atrial fibrillation were significant independent risk factors for AVR, not the choice of prosthesis (Table 1).

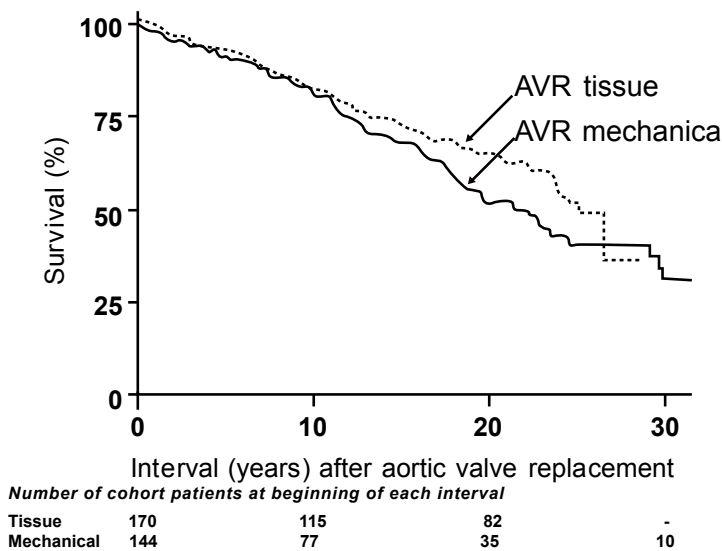

Figure 1: Kaplan-Meier survival curve for patients younger than 60 . Ruel et al. [13], with permission.

\begin{tabular}{|l|l|l|l|}
\hline & $\mathrm{HR}$ & $95 \% \mathrm{Cl}$ & $P$ \\
\hline Age at operation (per increasing year) & 1.03 & $1.01,1.04$ & 0.008 \\
\hline Coronary artery disease & 1.9 & $1.4,2.6$ & 0.002 \\
\hline Atrial fibrillation & 1.5 & $1.01,2.3$ & 0.04 \\
\hline Year of surgery (per increasing year) & 0.95 & $0.93,0.97$ & $<0.001$ \\
\hline Nonsignificant covariates & & & \\
\hline Female gender & 0.8 & $0.6,1.1$ & 0.2 \\
\hline Preoperative NYHA (per increasing year) & 1.2 & $0.98,1.3$ & 0.1 \\
\hline LV dysfunction $^{*}$ & 1.3 & $0.9,1.7$ & 0.1 \\
\hline Tissue prosthesis (VS mechanical) & 0.95 & $0.7,1.3$ & 0.7 \\
\hline $\begin{array}{l}\text { Contemporary } \\
\text { contemporary mechanical) }\end{array}$ & 1.2 & $0.7,2.0$ & 0.5 \\
\hline
\end{tabular}

* LV dysfunction = ejection fraction $<50 \%$

$\dagger$ Contemporary $=$ prosthesis are still commercially available

NYHA indicates New York Heart Association Functional Class; LV, left ventricular

Table 1: Predictors of late mortality after AVR in the study cohort, Ruel et al. [13], with permission. 
Other studies have determined that independent variables impacting long-term outcome were high NYHA class, concomitant CABG, and increasing age [21].

Operating on patients with multiple comorbidities and risk factors carries a high risk of morbidity and mortality. However, because medical therapy or balloon valvuloplasty are not acceptable alternatives to surgery, surgical options must be explored for these patients [22]. These patients account for a $22 \%$ of all patients in the US with Aortic Stenosis; they are deemed as ineligible for surgery when the risk of surgery is judged against the potential for improved survival and symptomatology [23]. Percutaneous deployment of an aortic valve via Transcatheter Aortic Valve Implantation (TAVI) technologies is a novel, less invasive, and widely successful alternative to surgical aortic valve replacement (Figure 2). This self-expanding pericardial bioprosthetic valve sits in a nitinol frame and is delivered via a percutaneous catheter through the femoral artery (transfemoral), or under general anesthesia by surgically exposing the apex of the left ventricle (transapical), the axillary or subclavian arteries, or the distal ascending aorta [23]. Currently there are two approved catheter valve delivery systems in clinical use - the SAPIEN valve (Edwards Lifesciences Inc.) and the CoreValve Revalving System (Medtronic Inc.). The SAPIEN valve utilizes a bovine pericardial bioprosthetic valve mounted onto a balloon-expandable stent which deploys the valve along the length of the native aortic valve. The CoreValve system deploys a self-expanding porcine pericardial valve. Both are delivered using an 18 French catheter system, on a stent which extends into the aortic root to anchor the deployed valve.

The transfemoral approach is the most widely used technique because of its ease of access, virtual absence of postoperative pain, and tolerance [23]. However, this approach is limited due to a high local complication at the puncture site and the need for relatively healthy vasculature; it is currently suitable for only low-profile device and delivery systems. Patients with severe peripheral vascular disease, tortuous iliac arteries, aortic aneurysms and extensive atheromas are not candidates for this approach. TAVI requires non-invasive imaging of the aortic annulus, echocardiography in particular, to determine eligibility and ideal implant size for patients [24]. Alternatively, the transapical approach allows direct access to the target location with antegrade crossing of the diseased valve. It also permits largersize delivery systems and avoids peripheral vascular complications. However, it does require a thoracotomy and complications may be more difficult to manage than via standard transfemoral techniques [23].

TAVI devices have shown immense success in the clinical realm, and studies have demonstrated successful transcatheter AVR via both the transfemoral and transapical approaches in thousands of patients [23]. In the PARTNER Trial, conducted at 21 centers worldwide, a total of 358 AS patients who were not candidates for SAVR were randomized to TAVI with the SAPIEN valve versus standard therapy (medical, possible balloon valvuloplasty). These patients had severe AS (defined as an aortic-valve area of $<0.8 \mathrm{~cm}^{2}$, mean AV gradient of $>40 \mathrm{mmHg}$, peak aortic-jet velocity of $>4 \mathrm{~m} / \mathrm{s}$ ), NYHA class II-IV symptoms, and were at high risk of death or morbidity for surgical therapy [25]. However, exclusion criteria included patients with bicuspid or noncalcified valves, post-acute myocardial infarction, coronary artery disease requiring revascularization, a left ventricular $\mathrm{EF}<20 \%$, aortic annular diameter $<18 \mathrm{~mm}$ or $>25 \mathrm{~mm}$, severe AR/MR ( $>3+)$, transient ischemic attack or stroke within the past 6 months, and severe renal insufficiency [25]. The authors found that at 1 year, death from any cause was $30.7 \%$ for TAVI versus $50.7 \%$ for standard therapy (HR 0.55 ;
95\% [CI] 0.4-0.74; $p<0.001)$. Furthermore, the composite end point of any-cause death or repeat hospitalization was $42.5 \%$ with TAVI versus $71.6 \%$ with standard therapy (HR 0.46; 95\% [CI] 0.35-0.59; $p<0.001$ ) (Figure 3). In conclusion, transfemoral TAVI was demonstrated to be superior to standard therapy via reduction of any-cause mortality, cardiovascular mortality, and repeat hospitalization. The authors also found a significant reduction in symptoms by assessing NYHA symptomatology and using results from a 6-minute walk test (Figure
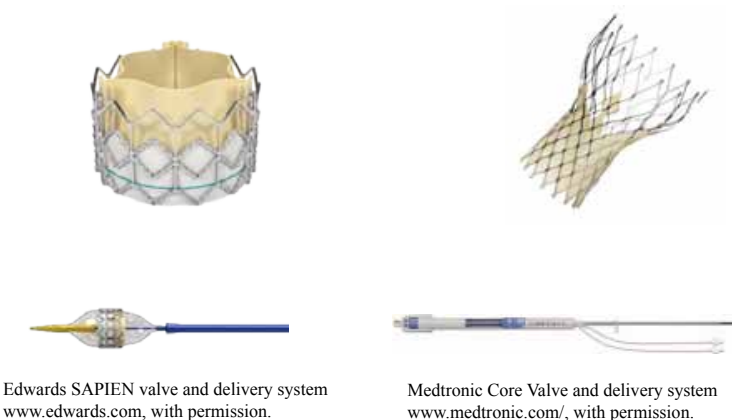
www.edwards.com, with permission. Medtronic Core Valve and delivery syste
www.medtronic.com/, with permission.

Figure 2: Percutaneous aortic valves with delivery systems.

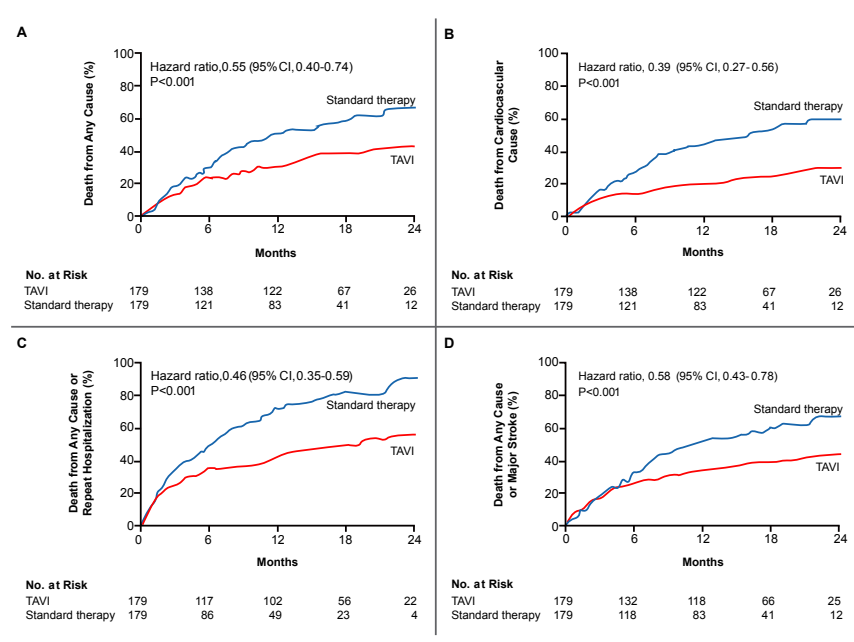

Figure 3: Comparison of mortality rates for TAVI and standard therapy. Leon et al. [25], with permission.

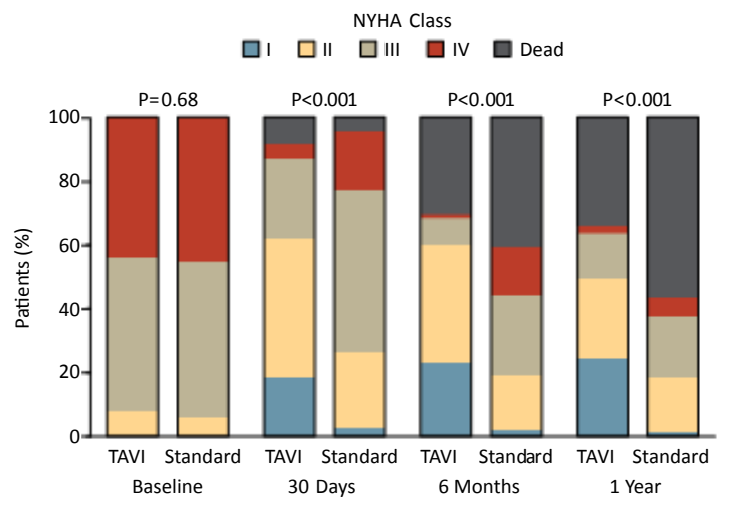

Figure 4: Patient symptoms at follow-up for TAVI vs. standard therapy. Leon et al. [25], with permission. 
4). However, there were more neurologic events (all strokes), major vascular complications, and major bleeding events in the TAVI group as compared to the standard-therapy group [25]. Reynolds et al. [26] demonstrated markedly improved survival and re-hospitalization rates at 2 years follow up for TAVI patients versus medical therapy.

The PARTNER trial investigators were the first to compare surgical AVR against TAVI in their randomized control trial [27] (Figure 5). Another group of 699 high-risk AS patients - as determined by a surgeon as well as with a Society of Thoracic Surgeons risk model score of at least $10 \%$ - were randomized to either SAVR or TAVI with the SAPEIN valve. The study was also stratified to transfemoral or transapical access based on the adequacy of the femoral arterial anatomy. For intentionto-treat analysis, the primary endpoints, or any-cause mortality after randomization, were $3.4 \%$ in the transcatheter group and $6.5 \%$ in the surgical group at 30 days $(\mathrm{P}=0.07)$ and $24.2 \%$ and $26.8 \%$, respectively, at 1 year $(\mathrm{P}=0.44$, non-inferiority $\mathrm{p}=0.001)$. The "as treated" transarterial AVR mortality was less than one half that of SAVR (3.7\% versus $8.2 \%)$, which was found to be statistically significant $(p=0.046)$. For patients undergoing transfemoral AVR, the mortality rates at 30 days and at 1 year in the TAVI group were $3.3 \%$ and $22.2 \%$, respectively, compared to $6.2 \%$ and $26.4 \%$ in the SAVR group ( $\mathrm{p}=0.13$ for 30 -day mortality, $\mathrm{p}=0.29$ for 1 -year mortality, non-inferiority $\mathrm{p}=0.002)$. The rates of major stroke were similar in both groups, at $3.8 \%$ and $2.1 \%(\mathrm{P}=0.20)$ at 30 days, and $5.1 \%$ and $2.4 \%$ at 1 year $(\mathrm{P}=0.07)$, respectively. Furthermore, there were no differences in stroke between transfemoral or transapical valve implantation techniques. However, major vascular complications were significantly higher with TAVI, with concerns about stroke rates, while major bleeding and new-onset atrial fibrillation were significantly higher with surgical AVR. With regards to clinical status, patients reported improved symptoms at 30 days in the TAVI group, but this was non-significant at 1 year when compared to surgical patients [27] (Figure 6). However, subgroup analysis of patients undergoing transapical TAVI revealed a higher rate of death at 30 days compared to transfemoral placement. This difference may be attributed to significantly increased rates of previous CABG, cerebrovascular, renal, and peripheral vascular disease in the patient population undergoing transapical TAVI. Furthermore, this difference may point to the steep learning curve required for trans-apical implantation.

While the results of the PARTNER trial demonstrated non-

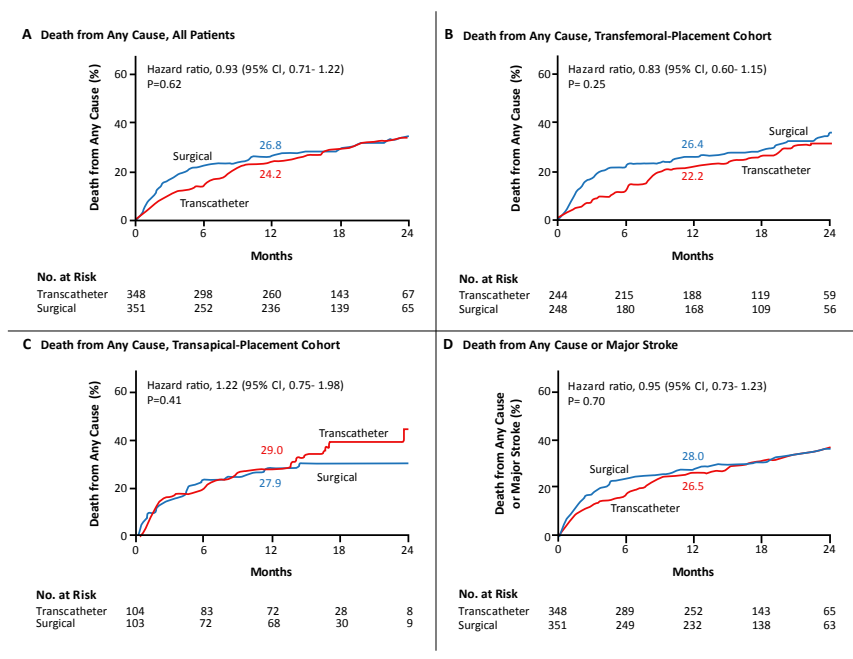

Figure 5: Comparison of mortality rates for TAVI and surgical AVR. Smith et al [26], with permission.

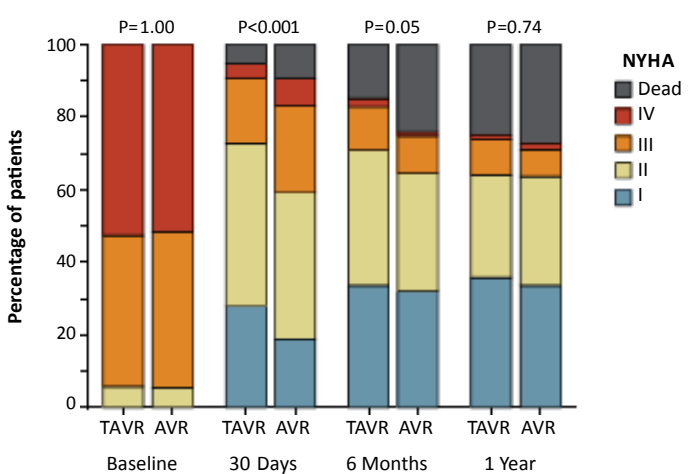

Figure 6: Patient symptoms at follow-up for TAVI vs surgical AVR. Smith et al. [26], with permission.

inferiority of TAVI compared to SAVR in a specific high-risk population of patients, the long-term results need to be evaluated. Previous studies by Clavel et al. [28] have outlined the benefits of TAVI over SAVR with regards to recovery of patients with poor left ventricular function $(\mathrm{EF}<50 \%)$. In this study, the authors found that at 1-year follow-up, $58 \%$ of TAVI patients had normalization of their ejection fraction versus $20 \%$ of those undergoing SAVR. Furthermore, risk factors in their study found to be associated with better LVEF recovery included female gender $(P=0.004)$, lower baseline LVEF $(P=0.005)$, absence of atrial fibrillation $(P=0.01)$, TAVI $(P=0.007)$, and a larger increase in aortic valve area after the procedure $(P=0.01)$ [28].

It has been demonstrated that TAVI is a successful, less-invasive alternative to surgical aortic valve replacement in specific populations. While TAVI has a myriad of benefits - which include but are not limited to improved symptomatology, mortality, and increased ejection fraction - we must be cognizant of its limitations. Data for long-term durability, mortality and morbidity benefit is lacking and currently under investigation. While paravalvular leaks are more commonly found in TAVI when compared to SAVR, most leaks are not clinically significant [29]. Furthermore, randomized control trials exclude a range of patients with pathologies such as bicuspid aortic valves or need for revascularization. Despite these limitations, patients have experienced an improvement in their functional status post-TAVI, with non-inferior mortality rates as compared to SAVR. This intervention is improving with increasing experience, patient selection, and technical improvements [29]. The limits for TAVI indications are being explored in a range of studies, including the PARTNER 2 and SURTAVI risk trials, to include patients who are in the highest $25 \%$ risk of patients [29]. Although the role of TAVI for aortic stenosis is extending to a larger population of patients, this will remain controversial for a period of time before outcome data is present. In conclusion, TAVI should be recommended as an alternative to SAVR for patients who are at highrisk of mortality and morbidity. However, patients selection for TAVI should be limited to patients who will likely benefit from an improved duration and quality of life despite their comorbidities.

Aortic stenosis affects a large population of patients and can cause substantial morbidity and mortality if left untreated. Treatment in a timely fashion is particularly important for symptomatic patients presenting with angina, syncope, or congestive heart failure. While open-heart surgical AVR is the current gold standard for treatment of aortic stenosis, percutaneous TAVI technology continues to play a key role in management of aortic stenosis in certain subpopulations of patients. 


\section{References}

1. Otto CM, Lind BK, Kitzman DW, Gersh BJ, Siscovick DS (1999) Association of aortic-valve sclerosis with cardiovascular mortality and morbidity in the elderly. N Engl J Med 341: 142-147.

2. Carabello BA (2002) Clinical practice. Aortic stenosis. N Engl J Med 346: 677682

3. Cripe L, Andelfinger G, Martin LJ, Shooner K, Benson DW (2004) Bicuspid aortic valve is heritable. J Am Coll Cardiol 44: 138-143.

4. Fernández B, Durán AC, Fernández MC, Arqué JM, Such M, et al. (2011) Genetic contribution of bicuspid aortic valve morphology. Am J Med Genet A 155: $2897-2898$

5. Cohn L (2007) Cardiac Surgery in the Adult ( $3^{\text {rd }}$ edn) Boston (MA): The McGraw-Hill Company.

6. American College of Cardiology; American Heart Association Task Force on Practice Guidelines (Writing Committee to revise the 1998 guidelines for the management of patients with valvular heart disease); Society of Cardiovascular Anesthesiologists, Bonow RO, Carabello BA, Chatterjee K, de Leon AC Jr, Faxon DP, et al. (2006) ACC/AHA 2006 guidelines for the management of patients with valvular heart disease: a report of the American College of Cardiology/American Heart Association Task Force on Practice Guidelines (writing Committee to Revise the 1998 guidelines for the management of patients with valvular heart disease) developed in collaboration with the Society of Cardiovascular Anesthesiologists endorsed by the Society for Cardiovascular Angiography and Interventions and the Society of Thoracic Surgeons. J Am Coll Cardiol 48: 1-148.

7. Braunwald E (2001) Valvular heart disease in Braunwald: Heart Disease: A Textbook of Cardiovascular Medicine. (6th edn), WB Saunders, New York (NY).

8. Lund O, Nielsen TT, Emmertsen K, Flo C, Rasmussen B, et al. (1996) Mortality and worsening of prognostic profile during waiting time for valve replacement in aortic stenosis. Thorac Cardiovasc Surg 44: 289-295.

9. Pibarot P, Dumesnil JG (2009) Prosthetic heart valves: selection of the optimal prosthesis and long-term management. Circulation 119: 1034-1048.

10. Hammermeister K, Sethi GK, Henderson WG, Grover FL, Oprian C, et al (2000) Outcomes 15 years after valve replacement with a mechanical versus a bioprosthetic valve: final report of the Veterans Affairs randomized trial. J Am Coll Cardiol 36: 1152-1158

11. Ruel M, Masters RG, Rubens FD, Bedard PJ, Pipe AL, et al. (2004) Late incidence and determinants of stroke after aortic and mitral valve replacement. Ann Thorac Surg 78: 77-83.

12. Briand M, Pibarot P, Després JP, Voisine P, Dumesnil JG, et al. (2006) Metabolic syndrome is associated with faster degeneration of bioprosthetic valves. Circulation 114: I512-517.

13. Ruel M, Chan V, Bédard P, Kulik A, Ressler L, et al. (2007) Very long-term survival implications of heart valve replacement with tissue versus mechanical prostheses in adults <60 years of age. Circulation 116: I294-300.

14. Akins CW, Buckley MJ, Daggett WM, Hilgenberg AD, Vlahakes GJ, et al. (1998) Risk of reoperative valve replacement for failed mitral and aortic bioprostheses. Ann Thorac Surg 65: 1545-1551.
15. Peterseim DS, Cen YY, Cheruvu S, Landolfo K, Bashore TM, et al. (1999) Long-term outcome after biologic versus mechanical aortic valve replacement in 841 patients. J Thorac Cardiovasc Surg 117: 890-897.

16. Jamieson WR, Burr LH, Miyagishima RT, Germann E, Macnab JS, et al. (2005) Carpentier-Edwards supra-annular aortic porcine bioprosthesis: clinical performance over 20 years. J Thorac Cardiovasc Surg 130: 994-1000.

17. Hanania G (2003) Which heart valve prosthesis for patients aged between 60 and 70 years? Heart 89: 481-482.

18. Emery RW, Krogh CC, Arom KV, Emery AM, Benyo-Albrecht K, et al. (2005) The St. Jude Medical cardiac valve prosthesis: a 25-year experience with single valve replacement. Ann Thorac Surg 79: 776-782.

19. Kulik A, Bedard P, Lam BK, Rubens FD, Hendry PJ, et al. (2006) Mechanica versus bioprosthetic valve replacement in middle-aged patients. Eur $\mathrm{J}$ Cardiothorac Surg 30: 485-491.

20. Ruel M, Kulik A, Lam BK, Rubens FD, Hendry PJ, et al. (2005) Long-term outcomes of valve replacement with modern prostheses in young adults. Eur $J$ Cardiothorac Surg 27: 425-433.

21. Ikonomidis JS, Kratz JM, Crumbley AJ 3rd, Stroud MR, Bradley SM, et al. (2003) Twenty-year experience with the St Jude Medical mechanical valve prosthesis. J Thorac Cardiovasc Surg 126: 2022-2031.

22. Warnes CA, Williams RG, Bashore TM, Child JS, Connolly HM, et al. (2008) ACC/AHA 2008 guidelines for the management of adults with congenital heart disease: a report of the American College of Cardiology/American Heart Association Task Force on Practice Guidelines (Writing Committee to Develop Guidelines on the Management of Adults With Congenital Heart Disease) Developed in Collaboration With the American Society of Echocardiography, Heart Rhythm Society, International Society for Adult Congenital Heart Disease, Society for Cardiovascular Angiography and Interventions, and Society of Thoracic Surgeons. J Am Coll Cardiol 52: 143-263.

23. Huber C, Feldman T (2010) Transcatheter Valve Therapies. New York (NY) Informa Healthcare.

24. Jayasuriya C, Moss RR, Munt B (2011) Transcatheter aortic valve implantation in aortic stenosis: The role of echocardiography. J Am Soc Echocardiogr 24 $15-27$

25. Leon MB, Smith CR, Mack M, Miller DC, Moses JW, et al. (2010) Transcatheter aortic-valve implantation for aortic stenosis in patients who cannot undergo surgery. N Engl J Med 363: 1597-1607.

26. Reynolds MR, Magnuson EA, Lei Y, Leon MB, Smith CR, et al. (2011) Healthrelated quality of life after transcatheter aortic valve replacement in inoperable patients with severe aortic stenosis. Circulation 124: 1964-1972.

27. Smith CR, Leon MB, Mack MJ, Miller DC, Moses JW, et al. (2011) Transcathete versus surgical aortic-valve replacement in high-risk patients. $\mathrm{N}$ Engl $\mathrm{J}$ Med 364: $2187-2198$

28. Clavel MA, Webb JG, Rodés-Cabau J, Masson JB, Dumont E, et al. (2010) Comparison between transcatheter and surgical prosthetic valve implantation in patients with severe aortic stenosis and reduced left ventricular ejection fraction. Circulation 122: 1928-1936.

29. Webb JG (2012) Mid-term follow-up after transcatheter aortic valve implantation. Eur Heart J 33: 947-948.
This article was originally published in a special issue, Cardiac Surgery: Aortic Valve Replacement and its Risk Factors handled by Editor(s). Dr. Cesario Bianchi, Brown Medical School, USA 